\title{
Intersection Theory
}


Springer Science+Business Media, LLC 
William Fulton

\section{Intersection Theory}

Second Edition 


\author{
William Fulton \\ Department of Mathematics \\ University of Michigan \\ Ann Arbor, MI 48109-1109 \\ USA \\ e-mail: fulton@math.lsa.umich.edu
}

Softcover Printing 1998 of the Second Edition 1998, which was originally published as Volume 2 of the series Ergebnisse der Mathematik und ihrer Grenzgebiete, 3. Folge.

Mathematics Subject Classification (1991): 14C17, 14-02, 14C10, 14C15, 14C25, 14C40, 14E10, 14M12, 14M15, 14N10, 55N45

Library of Congress Cataloging-in-Publication Data is on file

Printed on acid-free paper.

(C) 1998, 1984 Springer Science+Business Media New York

Originally published by Springer-Verlag New York, Inc. in 1998

All rights reserved. This work may not be translated or copied in whole or in part without the written permission of the publisher Springer Science+Business Media, LLC except for brief excerpts in connection with reviews or scholarly analysis. Use

in connection with any form of information storage and retrieval, electronic adaptation, computer software, or by similar or dissimilar methodology now known or hereafter developed is forbidden. The use of general descriptive names, trade names, trademarks, etc., in this publication, even if the former are not especially identified, is not to be taken as a sign that such names, as understood by the Trade Marks and Merchandise Marks Act, may accordingly be used freely by anyone.

Production managed by Bill Imbornoni; manufacturing supervised by Jacqui Ashri. 


\section{Preface to the Second Edition}

We thank P. Aluffi, P. Belorousski, G. Ellingsrud, L. van Gastel, H. Gillet, B. Gross, G. Kennedy, S. Kimura, S. Kleiman, K. Kurano, K. F. Lai, F. Oort, D. Perkinson, D. Ramakrishnan, W. Raskind, N. Ring, M. Saito, C. Soulé, H. Tamvakis, A. Vistoli, W. Vogel, S. Xambó, and some anonymous critics for supplying corrections.

No attempt has been made to survey the many developments in intersection theory since 1983 , other than adding some references which appeared not long after first edition. A few indications to more recent literature, as well as an informal introduction to the main ideas of this book, can be found in the 1996 edition of the author's Introduction to Intersection Theory in Algebraic Geometry, CBMS 57, Amer. Math. Soc., 1984, 1996.

October, 1997

William Fulton 


\section{Preface to the First Edition}

From the ancient origins of algebraic geometry in the solution of polynomial equations, through the triumphs of algebraic geometry during the last two centuries, intersection theory has played a central role. Since its role in foundational crises has been no less prominent, the lack of a complete modern treatise on intersection theory has been something of an embarrassment. The aim of this book is to develop the foundations of intersection theory, and to indicate the range of classical and modern applications. Although a comprehensive history of this vast subject is not attempted, we have tried to point out some of the striking early appearances of the ideas of intersection theory.

Recent improvements in our understanding not only yield a stronger and more useful theory than previously available, but also make it possible to develop the subject from the beginning with fewer prerequisites from algebra and algebraic geometry. It is hoped that the basic text can be read by one equipped with a first course in algebraic geometry, with occasional use of the two appendices. Some of the examples, and a few of the later sections, require more specialized knowledge. The text is designed so that one who understands the constructions and grants the main theorems of the first six chapters can read other chapters separately. Frequent parenthetical references to previous sections are included for such readers. The summaries which begin each chapter should facilitate use as a reference.

Several theorems are new or stronger than those which have appeared before, and some proofs are significantly simpler. Among the former are a new blow-up formula, a stronger residual intersection formula, and the removal of a projective hypotheses from intersection theory and Riemann-Roch theorems; the latter includes the proof of the Grothendieck-Riemann-Roch theorem. Some formulas from classical enumerative geometry receive a first modern or rigorous proof here.

Acknowledgements. The intersection theory described here was developed together with R. MacPherson. The author whose name appears on the cover is responsible for the presentation of details, and many of the applications and examples, but the extent to which it forms a coherent theory derives from collaboration with MacPherson. Previously unpublished results of R. Lazarsfeld, and joint work with Lazarsfeld, and with $\mathrm{H}$. Gillet, is also included. During the course of the work, many helpful suggestions were made by A. Collino, P. Deligne, S. Diaz, J. Harris, B. Iversen, S. L. Kleiman, A. Landman, Lazarsfeld, and J-P. Serre. Although other contributions and historical precedents are acknowledged in the text, many others, such as those of students and others who have responded to talks on these subjects, must be silently, but gratefully, cited. 
This undertaking was made possible by the support of several foundations and institutions. The Guggenheim Foundation provided a fellowship in 1980-81, the Sloan Foundation provided support in 1981-82, and grants have been received from the National Science Foundation during six years of research and writing on this subject. The support and hospitality of several institutions and their staffs has been equally vital: Mathematisk Institut of the University of Århus, Denmark (1976-77); Institute des Hautes Études Scientifiques, Bures-sur-Yvette, France (1981); Institute for Advanced Study, Princeton (1981-82); and Brown University. A summer course in Cortona, Italy in 1980 provided a chance to test a preliminary version of the first portion of the book. Thanks are due to the staffs at the IAS and Brown, especially to K. Jacques, for expert typing, and to the publishers for their cooperation. 


\section{Contents}

Introduction . . . . . . . . . . . . . . . . . 1

Chapter 1. Rational Equivalence . . . . . . . . . . . . . . . . . . 6

Summary . . . . . . . . . . . . . . . . . . . . . . . 6

1.1 Notation and Conventions . . . . . . . . . . . . . . . . 6

1.2 Orders of Zeros and Poles . . . . . . . . . . . . . . . . . 8

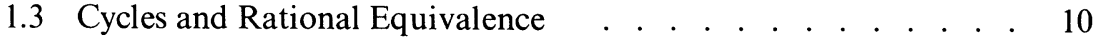

1.4 Push-forward of Cycles . . . . . . . . . . . . . . . . . . 11

1.5 Cycles of Subschemes . . . . . . . . . . . . . . . . . . 15

1.6 Alternate Definition of Rational Equivalence . . . . . . . . 15

1.7 Flat Pull-back of Cycles . . . . . . . . . . . . . . . . . 18

1.8 An Exact Sequence . . . . . . . . . . . . . . . . . . . . . . 21

1.9 Affine Bundles . . . . . . . . . . . . . . . . . . . . . . 22

1.10 Exterior Products . . . . . . . . . . . . . . . . . . . . . 24

Notes and References . . . . . . . . . . . . . . . . . . 25

Chapter 2. Divisors . . . . . . . . . . . . . . . . . . . . . . . 28

Summary . . . . . . . . . . . . . . . . . . . . . . . . . . 28

2.1 Cartier Divisors and Weil Divisors . . . . . . . . . . . . . 29

2.2 Line Bundles and Pseudo-divisors . . . . . . . . . . . . . . 31

2.3 Intersecting with Divisors . . . . . . . . . . . . . . . . . 33

2.4 Commutativity of Intersection Classes . . . . . . . . . . . . . 35

2.5 Chern Class of a Line Bundle . . . . . . . . . . . . . . . 41

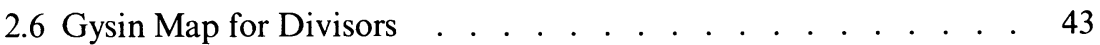

Notes and References . . . . . . . . . . . . . . . . . . . . 45

Chapter 3. Vector Bundles and Chern Classes _ . . . . . . . . . . . 47

Summary . . . . . . . . . . . . . . . . . . . . . . . . . . 47

3.1 Segre Classes of Vector Bundles _ . . . . . . . . . . . . . 47

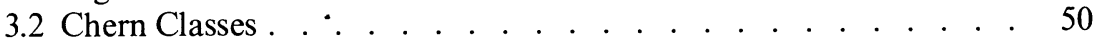

3.3 Rational Equivalence on Bundles . . . . . . . . . . . . . . 64

Notes and References . . . . . . . . . . . . . . . . . . . . . 68

Chapter 4. Cones and Segre Classes C . . . . . . . . . . . . . . . 70

Summary . . . . . . . . . . . . . . . . . . . . . . 70

4.1 Segre Class of a Cone . . . . . . . . . . . . . . . . . . 70 
4.2 Segre Class of a Subscheme . . . . . . . . . . . . . . . . 73

4.3 Multiplicity Along a Subvariety . . . . . . . . . . . . . . 79

4.4 Linear Systems . . . . . . . . . . . . . . . . . . . . . . 82

Notes and References . . . . . . . . . . . . . . . . . . . 85

Chapter 5. Deformation to the Normal Cone . . . . . . . . . . . 86

Summary . . . . . . . . . . . . . . . . . . . . . . . 86

5.1 The Deformation . . . . . . . . . . . . . . . . . . . 86

5.2 Specialization to the Normal Cone . . . . . . . . . . . . 89

Notes and References . . . . . . . . . . . . . . . . . . . . . 90

Chapter 6. Intersection Products . . . . . . . . . . . . . . . . . . 92

Summary . . . . . . . . . . . . . . . . . . . . . 92

6.1 The Basic Construction . . . . . . . . . . . . . . . . . . 93

6.2 Refined Gysin Homomorphisms . . . . . . . . . . . . . . 97

6.3 Excess Intersection Formula . . . . . . . . . . . . . . . . 102

6.4 Commutativity . . . . . . . . . . . . . . . . 106

6.5 Functoriality . . . . . . . . . . . . . . . . . . . . . . . 108

6.6 Local Complete Intersection Morphisms . . . . . . . . . . . . 112

6.7 Monoidal Transforms . . . . . . . . . . . . . . . . . . . 114

Notes and References . . . . . . . . . . . . . . . . . . 117

Chapter 7. Intersection Multiplicities . . . . . . . . . . . . . . . . 119

Summary . . . . . . . . . . . . . . . . . . . . . . 119

7.1 Proper Intersections . . . . . . . . . . . . . . . . . . . . 119

7.2 Criterion for Multiplicity One . . . . . . . . . . . . . . . 126

Notes and References . . . . . . . . . . . . . . . . . . 127

Chapter 8. Intersections on Non-singular Varieties . . . . . . . . . . 130

Summary . . . . . . . . . . . . . . . . . . . . . 130

8.1 Refined Intersections . . . . . . . . . . . . . . . . . . . . 130

8.2 Intersection Multiplicities . . . . . . . . . . . . . . . . . 137

8.3 Intersection Ring . . . . . . . . . . . . . . . . . . . . . 140

8.4 Bézout's Theorem (Classical Version) . . . . . . . . . . . . 144

Notes and References . . . . . . . . . . . . . . . . . . . . 151

Chapter 9. Excess and Residual Intersections . . . . . . . . . . . . 153

Summary . . . . . . . . . . . . . . . . . . . . . 153

9.1 Equivalence of a Connected Component . . . . . . . . . . . 153

9.2 Residual Intersection Theorem . . . . . . . . . . . . . . . 160

9.3 Double Point Formula . . . . . . . . . . . . . . . . . . . 165

Notes and References . . . . . . . . . . . . . . . . . . . . . $171^{\circ}$ 
Chapter 10. Families of Algebraic Cycles . . . . . . . . . . . . . 175

Summary . . . . . . . . . . . . . . . . . . . . . 175

10.1 Families of Cycle Classes . . . . . . . . . . . . . . . . 176

10.2 Conservation of Number . . . . . . . . . . . . . . . . . 180

10.3 Algebraic Equivalence . . . . . . . . . . . . . . . . . . 185

10.4 An Enumerative Problem . . . . . . . . . . . . . . . . . . . . . . 187

Notes and References . . . . . . . . . . . . . . . . . . . . . 193

Chapter 11. Dynamic Intersections . . . . . . . . . . . . . . . 195

Summary . . . . . . . . . . . . . . . . . . . . . . . . 195

11.1 Limits of Intersection Classes . . . . . . . . . . . . . . . 196

11.2 Infinitesimal Intersection Classes . . . . . . . . . . . . . . 198

11.3 Limits and Distinguished Varieties . . . . . . . . . . . . . 200

11.4 Moving Lemmas . . . . . . . . . . . . . . . . . . . . . 205

Notes and References . . . . . . . . . . . . . . . . . . . . . 209

Chapter 12. Positivity . . . . . . . . . . . . . . . . . . 210

Summary . . . . . . . . . . . . . . . . . . . 210

12.1 Positive Vector Bundles . . . . . . . . . . . . . . . . . . 211

12.2 Positive Intersections . . . . . . . . . . . . . . . . . . . 218

12.3 Refined Bézout Theorem . . . . . . . . . . . . . . . . . 223

12.4 Intersection Multiplicities . . . . . . . . . . . . . . . . . 227

Notes and References . . . . . . . . . . . . . . . . . . . 234

Chapter 13. Rationality . . . . . . . . . . . . . . . . . . 235

Summary . . . . . . . . . . . . . . . . . . . . 235

Notes and References . . . . . . . . . . . . . . . . . 241

Chapter 14. Degeneracy Loci and Grassmannians . . . . . . . . . . 242

Summary . . . . . . . . . . . . . . . . . . . . . . . . . . 242

14.1 Localized Top Chern Class . . . . . . . . . . . . . . . . 244

14.2 Gysin Formulas . . . . . . . . . . . . . . . . . . . . . 247

14.3 Determinantal Formula . . . . . . . . . . . . . . . . 249

14.4 Thom-Porteous Formula . . . . . . . . . . . . . . . . 254

14.5 Schur Polynomials . . . . . . . . . . . . . . . . . . 263

14.6 Grassmann Bundles . . . . . . . . . . . . . . . . . . . . 266

14.7 Schubert Calculus . . . . . . . . . . . . . . . . . 271

Notes and References . . . . . . . . . . . . . . . . . . 278

Chapter 15. Riemann-Roch for Non-singular Varieties . . . . . . . . 280

Summary . . . . . . . . . . . . . . . . . . . . 280

15.1 Preliminaries. . . . . . . . . . . . . . . . . . . . . . . 280

15.2 Grothendieck-Riemann-Roch Theorem . . . . . . . . . . . 286

15.3 Riemann-Roch Without Denominators . . . . . . . . . . . 296 
15.4 Blowing up Chern Classes . . . . . . . . . . . . . . . 298

Notes and References . . . . . . . . . . . . . . . . . . . . . 302

Chapter 16. Correspondences . . . . . . . . . . . . . . . . . . . 305

Summary . . . . . . . . . . . . . . . . . . . 305

16.1 Algebra of Correspondences . . . . . . . . . . . . 305

16.2 Irregular Fixed Points . . . . . . . . . . . . . . . . . . 315

Notes and References . . . . . . . . . . . . . . . . . . . . 318

Chapter 17. Bivariant Intersection Theory _ . . . . . . . . . . . . 319

Summary . . . . . . . . . . . . . . . . . . . . . . . . 319

17.1 Bivariant Rational Equivalence Classes . . . . . . . . . . . 320

17.2 Operations and Properties . . . . . . . . . . . . . . . . . 322

17.3 Homology and Cohomology . . . . . . . . . . . . . . . . 324

17.4 Orientations . . . . . . . . . . . . . . . . . . 326

17.5 Monoidal Transforms . . . . . . . . . . . . . . . . . . . 332

17.6 Residual Intersection Theorem . . . . . . . . . . . . . . . 333

Notes and References . . . . . . . . . . . . . . . . . . . 337

Chapter 18. Riemann-Roch for Singular Varieties . . . . . . . . . . . 339

Summary . . . . . . . . . . . . . . . . . . . . 339

18.1 Graph Construction . . . . . . . . . . . . . . . . . . . 340

18.2 Riemann-Roch for Quasi-projective Schemes . . . . . . . . 348

18.3 Riemann-Roch for Algebraic Schemes . . . . . . . . . . . 353

Notes and References . . . . . . . . . . . . . . . . . . . . . 368

Chapter 19. Algebraic, Homological and Numerical Equivalence . . 370

Summary . . . . . . . . . . . . . . . . . . . . . . . 370

19.1 Cycle Map . . . . . . . . . . . . . . . . . . . . . . . 371

19.2 Algebraic and Topological Intersections . . . . . . . . . 378

19.3 Equivalence on Non-singular Varieties . . . . . . . . . . . 385

Notes and References . . . . . . . . . . . . . . . . . . . . . 391

Chapter 20. Generalizations . . . . . . . . . . . . . . . . . . . . 393

Summary . . . . . . . . . . . . . . . . . . . . . . . . 393

20.1 Schemes Over a Regular Base Scheme . . . . . . . . . . 393

20.2 Schemes Over a Dedekind Domain . . . . . . . . . . . . 397

20.3 Specialization . . . . . . . . . . . . . . . . . . . . . . . 398

20.4 Tor and Intersection Products . . . . . . . . . . . . . . . 401

20.5 Higher $K$-theory . . . . . . . . . . . . . . . . . . . . . . 403

Notes and References . . . . . . . . . . . . . . . . . . . . . 404

Appendix A. Algebra . . . . . . . . . . . . . . . . . . . . . . 406

Summary . . . . . . . . . . . . . . . . . . 406

A.1 Length. . . . . . . . . . . . . . . . . . . . 406 
A.2 Herbrand Quotients . . . . . . . . . . . . . . . . . . . . 407

A.3 Order Functions . . . . . . . . . . . . . . . . . . . . . 411

A.4 Flatness . . . . . . . . . . . . . . . . . . . . . . . . 413

A.5 Koszul Complexes . . . . . . . . . . . . . . . . . . . . 414

A.6 Regular Sequences . . . . . . . . . . . . . . . . . . . . 416

A.7 Depth . . . . . . . . . . . . . . . . . . . . . . . . 418

A.8 Normal Domains . . . . . . . . . . . . . . . . . . . . . 419

A.9 Determinantal Identities . . . . . . . . . . . . . . . . . . . . 419

Notes and References . . . . . . . . . . . . . . . . . . . . 425

Appendix B. Algebraic Geometry (Glossary) . . . . . . . . . . . . 426

B.1 Algebraic Schemes . . . . . . . . . . . . . . . . . 426

B.2 Morphisms . . . . . . . . . . . . . . . . . . . 427

B.3 Vector Bundles . . . . . . . . . . . . . . . . . . . . . . 430

B.4 Cartier Divisors . . . . . . . . . . . . . . . . . . . . . . 431

B.5 Projective Cones and Bundles . . . . . . . . . . . . . . . 432

B.6 Normal Cones and Blowing Up . . . . . . . . . . . . . . . 435

B.7 Regular Imbeddings and l.c.i. Morphisms . . . . . . . . . . 437

B.8 Bundles on Imbeddable Schemes . . . . . . . . . . . . . . 439

B.9 General Position . . . . . . . . . . . . . . . . . . . . . 440

Bibliography . . . . . . . . . . . . . . . . . . . . 442

Notation . . . . . . . . . . . . . . . . . . . . . . 462

Index. . . . . . . . . . . . . . . . . . . . . . . . . . . 464 